\title{
Performance Tradeoffs between Maximum Ratio Transmission and Switched-Transmit Diversity
}

\author{
Ching-Hok Tse, Kun-Wah Yip and Tung-Sang Ng \\ \{chtse, kwyip, tsng\}@eee.hku.hk \\ Department of Electrical and Electronic Engineering \\ The University of Hong Kong \\ Pokfulam Road, Hong Kong \\ Fax: $++852+25598738$ \\ Tel.: $++852+28578406$
}

\begin{abstract}
Implementation of maximum ratio transmission (MRT) systems is not a trivial task. Alternatively, switched-transmitter diversity (STD) systems are less difficult to implement, but their performance is suboptimal when compared to MRT systems. If the difference is not significant, implementation advantages of STD systems outweigh the minor performance loss so that these systems are desirable for practical implementations. This paper compares the performances between MRT and STD systems under Rayleigh-fading conditions for $2 \times 1,2 \times 2$ and $4 \times$ (transmit antennas $\times$ receive antennas) configurations. It is found that the differences are only about $1 \mathrm{~dB}$ for the $2 \times 1$ and $2 \times 2$ configurations, indicating that implementation simplicity of a STD system can be utilized without sacrificing too much performance. However, the difference is around $3 \mathrm{~dB}$ for the $4 \times$ configuration. The considerably larger loss prompts system designers to consider more critically on the tradeoffs between implementation aspects and performances of STD and MRT systems.
\end{abstract}

\section{INTRODUCTION}

For communication systems employing transmit- and/or receive-antenna diversity, Lo [1] has recently proposed the maximum ratio transmission (MRT) technique, which is based on maximization of the overall signal-to-noise ratio (SNR). The system model considered in [1] is a discrete-time model equivalent to the corresponding continuous-time communication system. A knowledge of an $L \times K$ transmission matrix is assumed available where $K$ and $L$ are the numbers of transmit and receive antennas, respectively. The $(\ell, k)$ th entry of this matrix models the signal gain of an information symbol transmitted via the $k$ th transmit antenna and acquired by the $\ell$ th receive antenna. This signal gain represents the combined effects of transmitter filtering, physical channel, receiver matched filtering and sampling of the corresponding communication system.

Since the transmission matrix is modeled by $L$ rows, one can construct the equivalent discrete-time model from the communication system only if the receiver produces exactly one output for each of the $L$ received signals. In addition, the model of [1] assumes that only $L$ outputs are combined together to form the decision variable. These two requirements are satisfied if the $K$ transmitted signals are time-synchronized. In this case, the $K$ signals are added coherently at a receive antenna so that the information symbol embedded in the $K$ signals can be recovered by using one matched filter. However, implementation of synchronous transmission among all branches is not a trivial task because of possible non-identical delays introduced by RF filters and amplifiers. In addition, the signal gains provided by the RF amplifiers are needed to be accurate. Linear amplifiers with controllable, accurate gains are rather expensive.

Alternatively, system designers may employ the switched-transmitter (ST) strategy to alleviate the difficulty encountered in the implementation of MRT systems. In a ST diversity (STD) system, transmission is entirely through the transmit antenna that has the best channel condition, and the rest of transmit antennas are

This work was supported by the Hong Kong Research Grants Council and by the University Research Committee of The University of Hong Kong, Hong Kong.

0-7803-6465-5/00\$10.00@ 2000 IEEE 
deactivated. Classical maximum ratio combing (MRC) may be used at the receiver for combing signals received at different receive antennas. Implementation considerations for STD systems have been investigated by Wittneben and Kaltenschnee [2], and Wittneben [3], [4]. Furthermore, Yip and $\mathrm{Ng}$ [5] have shown that a system using STD at the transmitter side and MRC at the receiver side is performance-equivalent to a MRT system (which maximizes the SNR) under the special condition that orthogonal signaling waveforms are used among different transmit branches.

It can be easily shown that a STD system can be modeled by the same system model employed in [1] but the solution to the assignment of signal power for transmit branches is different from the optimal solution as employed in MRT. Therefore, a STD system yields a suboptimal performance when compared to a MRT system. Because of implementation advantages of the STD system over the MRT system, it becomes useful to consider the performance difference between the two systems. If the difference is not significant, implementation advantages of STD systems outweigh the minor performance loss so that these systems are desirable for practical implementations. This performance-loss issue is the subject of investigation of this paper.

We focus our attention on system realizations based on a) 2 transmit and 1 receive antennas, b) 4 transmit and 1 receive antennas, and c) 2 transmit and 2 receive antennas. These realizations are denoted as $2 \times 1,4 \times$ and $2 \times 2$ antenna-diversity systems, respectively. Other realizations with higher number of antennas are less common and are not considered here. Simulation was used to obtain the numerical performance figures. Rayleigh-fading environments were considered. Since we wish to obtain insights in the performance difference between the MRT and the STD systems, we consider binary phase-shift keying (BPSK) for simplicity. Section II describes the simulation models. Simulation results are presented and discussed in Section III. Conclusions are also given.

\section{SIMULATION MODELS}

Commonalties between a MRT and a STD systems permit modeling these two systems by a similar system model. Without explicit statements and unless otherwise stated, the model that is to be presented is applicable for both systems. In the system model that is described, $K$ transmit and $L$ receive antennas are used. The system model is shown in Fig. 1. Furthermore, we denote that the $k$ th transmitted signal is the signal transmitted via the $k$ th transmit antennas. Similarly, the $\ell$ th received signal is the signal received at the $\ell$ th receive antenna. BPSK is considered.

\section{A. Transmitter model}

For the system using MRT, all $K$ transmitted signals are time-synchronized and the $k$ th transmitted signal before transmission is weighted by a gain $v_{k}$, which is determined based on the objective of maximizing the overall SNR. This MRT transmitter model can be carried over to model a STD system, provided that in a STD system only one of $v_{k}$ 's, $k=1,2, \ldots, K$, is nonzero. The complex envelop of the $k$ th transmitted BPSK signal (applicable to model both systems under consideration) is given by

$$
s_{k}(t)=\sqrt{2 P} v_{k} \sum_{m=-\infty}^{\infty} I_{m} \psi\left(t-m T_{b}\right)
$$

where $I_{m} \in\{+1,-1\}$ is the $m$ th transmitted bit, $P$ is the total transmitted power, $/ T_{b}$ is the bit transmission rate, and $(t)$ is the pulse shape satisfying $\int_{-\infty}^{\infty}|\psi(t)|^{2} d t=T_{b}$ and not arousing intersymbol interference. For a fixed total transmitted power $P$, it is required that

$$
\mathbf{v}^{\dagger} \mathbf{v}=1
$$

where

$$
\mathbf{v}=\left[v_{1}, v_{2}, \cdots, v_{K}\right]^{T}
$$

and $(.)^{\dagger}$ denotes complex-conjugate transpose.

\section{B. Channel and receiver models}

The complex envelop of the $\ell$ th received signal is given by

$$
r_{\ell}(t)=\eta_{\ell}(t)+\sum_{k=1}^{K} h_{\ell k} s_{k}(t)
$$

where $h_{\ell k}$ is the channel provided for the signal sent via the $k$ th transmit antenna and acquired by the $\ell$ th receive antenna, and $\eta_{\ell}(t)$ is the basebandequivalent additive white Gaussian noise (AWGN) having a one-sided power spectral density $N_{0}$. It is assumed that $\eta_{\ell}(t), \ell=1,2, \ldots, L$, are statistically independent. Rayleigh fading is considered for each of the $L K$ channels. The channels can be statistically independent or correlated. It is assumed that each channel has a unity gain, i.e., $E\left\{\left|h_{\ell k}\right|^{2}\right\}=1$, so that $P$ is the average bit energy of the signal received at a receive antenna. The signal $r_{\ell}(t)$ is processed by a matched filter. Assume that the $n$th bit is the desired bit to be recovered. The matched-filter output at the $n$th sampling instant, $\xi_{\ell, n}$, is given by 


$$
\begin{aligned}
\xi_{\ell, n} & =\frac{1}{\sqrt{2 P} T_{b}} \int_{-\infty}^{\infty} r_{\ell}(t) \psi^{*}\left(t-n T_{b}\right) d t \\
& =\eta_{\ell, n}+I_{n} \sum_{k=1}^{K} h_{\ell k} v_{k}
\end{aligned}
$$

where $\eta_{e n}$ is a zero-mean complex-Gaussian random variable with a variance $\left(E_{b} / N_{0}\right)^{-1}$. In the last expression, $E_{b}=P T_{b}$ is the bit energy. Let

$$
\begin{aligned}
& \xi_{n}=\left[\xi_{1, n}, \xi_{2, n}, \cdots, \xi_{L, n}\right]^{T}, \\
& \eta_{n}=\left[\eta_{1, n}, \eta_{2, n}, \cdots, \eta_{L, n}\right]^{T}
\end{aligned}
$$

and

$$
\mathbf{H}=\left[\begin{array}{cccc}
h_{11} & h_{12} & \cdots & h_{1 K} \\
h_{21} & h_{22} & & \vdots \\
\vdots & & \ddots & \vdots \\
h_{L 1} & h_{L 2} & \cdots & h_{L K}
\end{array}\right]
$$

It follows that

$$
\xi_{n}=\eta_{n}+I_{n} \mathbf{H v} .
$$

The $L$ matched-filter outputs are combined together to form the decision variable, $\hat{I}_{n}$, given by

$$
\hat{I}_{n}=\mathbf{w}^{T} \xi_{n}
$$

where

$$
\mathbf{w}=\left[w_{1}, w_{2}, \cdots, w_{L}\right]^{T}
$$

contains the weights.

\section{Selection of $\mathbf{v}$ and $\mathbf{w}$}

$M R T)$ Determination of the optimal $\mathbf{v}$ and $\mathbf{w}$ follows the lines of [6]. By the MRC principle, the overall SNR is maximized if

$$
\mathbf{w}^{T}=(\mathbf{H v})^{\dagger} .
$$

It follows that the SNR, $\gamma$, is given by

$$
\gamma=\left(E_{b} / N_{0}\right) \mathbf{v}^{\dagger} \mathbf{H}^{\dagger} \mathbf{H} \mathbf{v} .
$$

Maximizing $\gamma$ can be accomplished by choosing the optimal $\mathbf{v}$, denoted by $\mathbf{v}_{\mathrm{MRT}}$, that maximizes the quadratic form $\mathbf{v}^{\dagger} \mathbf{H}^{\dagger} \mathbf{H v}$ subject to the constraint $\mathbf{v}^{\dagger} \mathbf{v}=1$. Let $\gamma_{\text {MRT }}$ be the maximum achievable SNR, viz., $\gamma_{\mathrm{MRT}}=$ $\left(E_{b} / N_{0}\right) \mathbf{v}_{\mathrm{MRT}}^{\dagger} \mathbf{H}^{\dagger} \mathbf{H} \mathbf{v}_{\mathrm{MRT}}^{\dagger}$. It is known that $\mathbf{v}^{\dagger} \mathbf{H}^{\dagger} \mathbf{H v}$ can be maximized by finding the maximum eigenvalue of $\mathbf{H}^{\dagger} \mathbf{H}[7$, p. 220]. It yields

$$
\gamma_{\mathrm{MRT}}=\left(E_{b} / N_{0}\right) \lambda_{\max }
$$

and

$$
\mathbf{v}_{\text {MRT }}=\mathbf{u}_{\max }
$$

where $\lambda_{\max }$ and $\mathbf{u}_{\max }$ are the maximum eigenvalue and its associated orthonormal eigenvector, respectively, of the matrix $\mathbf{H}^{\dagger} \mathbf{H}$. The optimal combining-weight vector $w$, denoted by $\mathbf{w}_{\mathrm{MRT}}$, immediately follows from (12) and is given by

$$
\mathbf{w}_{\mathrm{MRT}}^{T}=\left(\mathbf{H} \mathbf{v}_{\mathrm{MRT}}\right)^{\dagger}=\left(\mathbf{H} \mathbf{u}_{\max }\right)^{\dagger} .
$$

STD) Among the $K$ transmit antennas, the one with the maximum channel gain is selected for transmission. The channel gain for the $k$ th transmit antenna, $\theta_{k}$, is given by

$$
\theta_{k}=\sum_{\ell=1}^{L}\left|h_{\ell k}\right|^{2}
$$

The transmit-gain vector for the STD system, denoted by $\mathbf{v}_{\mathrm{STD}}$, is given by

$$
\mathbf{v}_{\mathrm{STD}}=\left[\delta_{1 k^{\prime}}, \delta_{2 k^{\prime}}, \cdots, \delta_{K k^{\prime}}\right]^{T}
$$

where $\delta_{(\cdot)}$ is the Kroneckor delta function and $k^{\prime}=$ $\arg \max _{k^{\prime}=1,2, \cdots, K} \theta_{k^{\prime}}$. The combining weight, $\mathbf{w}_{\mathrm{STD}}$, also follows from (12), and is given by

$$
\mathbf{w}_{\text {STD }}^{T}=\left(\mathbf{H v}_{\text {STD }}\right)^{\dagger} \text {. }
$$

\section{NUMERICAL RESULTS AND CONCLUSIONS}

We consider the $2 \times 1,2 \times 2$ and $4 \times$ configurations. The cases of independent and correlated Rayleigh-fading channels are studied. In the case of correlated-fading channels, we model the correlation coefficients as follows. For the $2 \times 1$ configuration, $\rho$ is the correlation coefficient. For the $2 \times 2$ configuration, it is assumed that (i) $\rho$ is the correlation between adjacent transmit antennas (i.e.: $h_{11}$ and $h_{12} ; h_{21}$ and $h_{22}$ ) and also is the correlation between adjacent receive antennas (viz.: $h_{11}$ and $h_{21} ; h_{12}$ and $h_{22}$ ), and (ii) the correlation coefficient between a cross pair of transmit and receive antennas (i.e.: $h_{11}$ and $h_{22} ; h_{12}$ and $\left.h_{21}\right)$ is $\rho^{2}$. It follows that the covariance matrix is modeled by

$$
\operatorname{cov}\left(\left[h_{11}, h_{12}, h_{21}, h_{22}\right]\right)=\left[\begin{array}{cccc}
1 & \rho & \rho & \rho^{2} \\
\rho & 1 & \rho^{2} & \rho \\
\rho & \rho^{2} & 1 & \rho \\
\rho^{2} & \rho & \rho & 1
\end{array}\right]
$$

For the $4 \times$ configuration, we follow the same approach in modeling the correlation coefficients. We assign that (i) $\rho$ is the correlation between adjacent transmit antennas (i.e., antennas spaced by one unit of distance), (ii) the correlation is $\rho^{2}$ for antennas with two units of distance in separation, and (iii) the correlation is $\rho^{3}$ for those separated 
with three units of distance. It yields

$$
\operatorname{cov}\left(\left[h_{11}, h_{12}, h_{13}, h_{14}\right]\right)=\left[\begin{array}{cccc}
1 & \rho & \rho^{2} & \rho^{3} \\
\rho & 1 & \rho & \rho^{2} \\
\rho^{2} & \rho & 1 & \rho \\
\rho^{3} & \rho^{2} & \rho & 1
\end{array}\right]
$$

In the simulation, $\rho=0$ and $\rho=0.6$, modeling independent and correlated fading respectively, were assumed. A total of $0^{6}$ data points were simulated for each SNR value and for each antenna configuration.

Figs. 2, 3 and 4 plot the bit error rates (BERs) for the configurations $2 \times 1,2 \times 2$ and $4 \times$, respectively, for both cases of MRT and STD. It is apparent that the performance of MRT is better than that of STD, a result that is expected. Furthermore, it is shown that performances for both MRT and STD systems in the cases $\rho=0$ and $\rho=$ 0.6 are very close. For both cases of channel fading correlation, it is apparent from Figs. 2 and 3 that $\mathrm{dB}$ gains for MRT over STD at a BER of $0^{-3}$ are only about $1 \mathrm{~dB}$ for the $2 \times 1$ and $2 \times 2$ configurations. These $\mathrm{dB}$ gains are not very significant, indicating that one can take advantage of the implementation simplicity of a STD system without sacrificing too much performance. However, Fig. 4 indicates that the $\mathrm{dB}$ gain is around $3 \mathrm{~dB}$ for the $4 \times$ configuration. The considerably larger performance loss of a STD system in this configuration prompts system designers to consider more critically on the tradeoffs between implementation aspects and achievable performances of STD and MRT systems.

\section{REFERENCES}

[1] T. K. Y. Lo, "Maximum ratio transmission," IEEE Trans. Commun., vol. 47, pp. 1458-1461, Oct. 1999.

[2] A. Wittneben and T. Kaltenschnee, "TX selection diversity with prediction: systematic nonadaptive predictor design," Proc. IEEE VTC'94, pp. 1246-1250, Jun. 8-10, 1994.

[3] A. Wittneben, "Optimal predictive TX combining diversity in correlated fading for microcellular mobile radio applications," Proc. IEEE GLOBECOM'95, pp. 48-54, Nov. 13-17, 1995.

[4] A. Wittneben, "Analysis and comparison of optimal predictive transmitter selection and combining diversity for DECT," Proc. IEEE GLOBECOM'95, pp. 1527-1531, Nov. 13-17, 1995.

[5] K. W. Yip and T. S. Ng, "Optimal-weight selection for maximum ratio transmission using orthogonal waveforms," submitted to IEEE Trans. Commun.

[6] K. W. Yip and T. S. Ng, "Weight-vector optimization for maximum ratio transmission," submitted to IEEE Trans. Commun.

[7] F. Zhang, Matrix Theory: Basic Results and Techniques, Springer: New York, 1999. 
Fig. 1. System model.

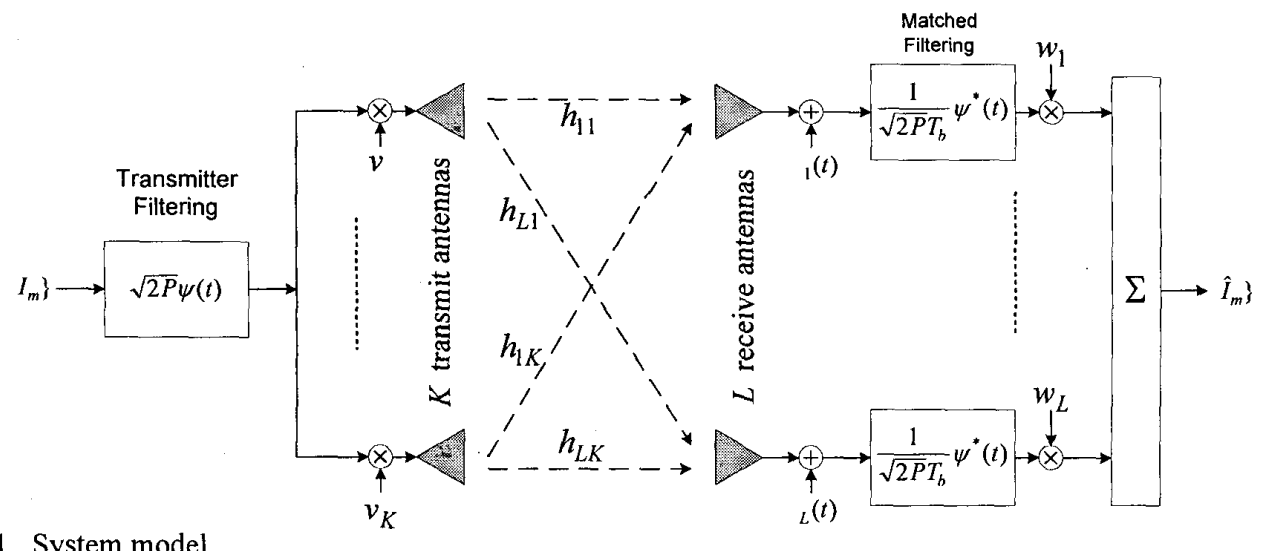

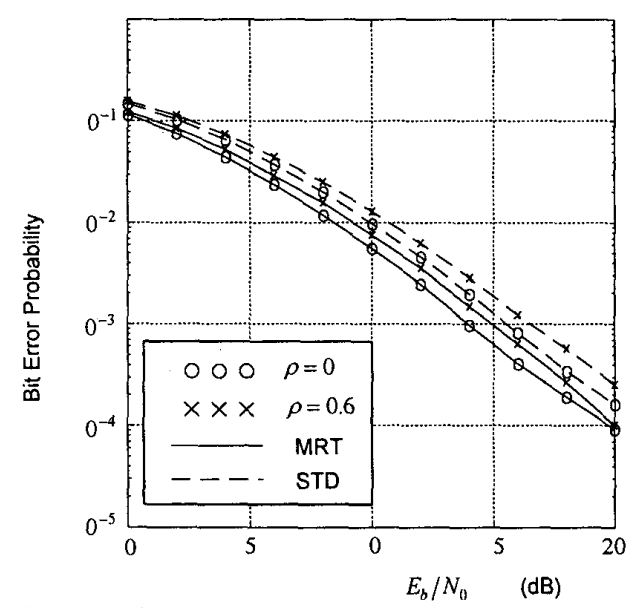

Fig. 2. Bit error rate for MRT and STD systems using $2 \times 1$ antenna diversity.

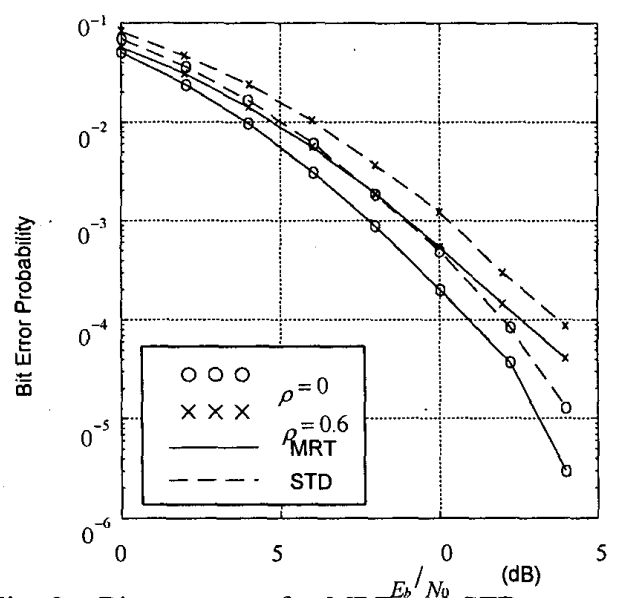

Fig. 3. Bit error rate for MRT and STD systems using $2 \times 2$ antenna diversity.

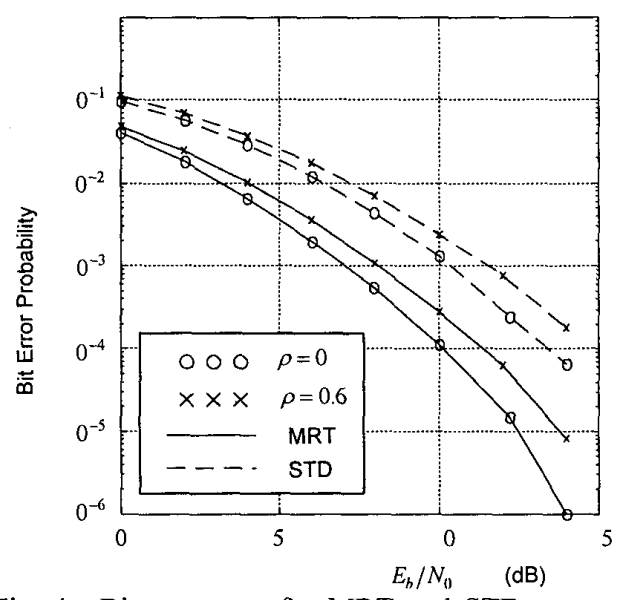

Fig. 4. Bit error rate for MRT and STD systems using $4 \times$ antenna diversity. 